\title{
Symptomatic and urodynamic responses in patients with reduced or no seminal emission during silodosin treatment for LUTS and BPH
}

\author{
CG Roehrborn ${ }^{1}$, SA Kaplan ${ }^{2}$, H Lepor ${ }^{3}$ and W Volinn ${ }^{4}$ \\ ${ }^{1}$ Department of Urology, University of Texas Southwestern Medical Center, Dallas, TX, USA; ${ }^{2}$ Department of Urology, Institute of \\ Bladder and Prostate Health, Weill College of Medicine, Cornell University, New York, NY, USA; ${ }^{3}$ Department of Urology, New York \\ University, New York, NY, USA and ${ }^{4}$ Clinical Research, Watson Laboratories, Salt Lake City, UT, USA
}

\begin{abstract}
Data from phase 3 studies (NCT00224107, NCT00224120) of silodosin for treatment of BPH symptoms were analyzed to examine the relationship between treatment efficacy and occurrence of abnormal ejaculation. Men aged $\geqslant 50$ years with International Prostate Symptom Scores (IPSS) $\geqslant 13$ and peak urinary flow rates (Qmax) of $4-15 \mathrm{ml} \mathrm{s}^{-1}$ received placebo or silodosin $8 \mathrm{mg}$ once daily for 12 weeks. Silodosin-treated patients were stratified by absence or presence of 'retrograde ejaculation' (RE). Groups were compared using analysis of covariance (for change from baseline) and responder analyses. Of the 466 patients receiving silodosin, $131(28 \%)$ reported RE and $335(72 \%)$ did not; 4 of the 457 patients receiving placebo $(0.9 \%)$ reported RE. Most RE events in silodosin-treated patients $(110 / 134 ; 82 \%)$ were reported as 'orgasm with absence of seminal emission.' Silodosin-treated patients with $(+)$ and without $(-)$ RE showed significant improvement in IPSS, Qmax and quality of life versus placebo $(P<0.02)$. RE + patients versus $R E$ - patients experienced numerically greater improvement, but differences were not statistically significant $(P>0.05)$. For $R E+$ patients, the odds of achieving improvement of $\geqslant 3$ points in IPSS and $\geqslant 3 \mathrm{ml} \mathrm{s}^{-1}$ in Qmax by study end were 1.75 times those for RE- patients $(P=0.0127)$. Absence of seminal emission may predict superior treatment efficacy of silodosin in individual patients. Prostate Cancer and Prostatic Diseases (2011) 14, 143-148; doi:10.1038/pcan.2010.46; published online 7 December 2010
\end{abstract}

Keywords: BPH; LUTS; $\alpha$-blocker; retrograde ejaculation; IPSS score

\section{Introduction}

Treatment with $\alpha$-blockers has been shown to relieve BPH-related symptoms and improve urinary flow. ${ }^{1,2}$ $\alpha$-Blockers are believed to act by inducing smooth muscle relaxation in the bladder neck, prostate and prostatic capsule through the blockade of $\alpha_{1}$-adrenergic receptors. A vast majority of $\alpha_{1}$-receptors in these tissues are thought to belong to the $\alpha_{1 \mathrm{a}}$-receptor subtype. ${ }^{1}$

The efficacy of providing symptom relief in patients with BPH-associated lower urinary tract symptoms (LUTS) is thought to be similar for all $\alpha$-blockers, regardless of their selectivity for $\alpha_{1}$-receptor subtypes,

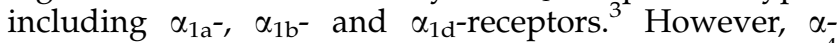
blockers differ in their safety and tolerability profiles, ${ }^{4}$ particularly in their propensity to cause abnormal ejaculation. ${ }^{5}$ In controlled clinical trials, the percentage of patients treated with the moderately $\alpha_{1 a}$-selective $\alpha$ blocker tamsulosin who reported abnormal ejaculation varied between 4 and $26 \%$, depending on dose and study

Correspondence: Dr CG Roehrborn, University of Texas Southwestern Medical Center, Department of Urology, 5323 Harry Hines Boulevard, J8.148, Dallas, TX 75390-9910, USA.

E-mail: claus.roehrborn@utsouthwestern.edu

Received 6 June 2010; revised 13 September 2010; accepted 20

September 2010; published online 7 December 2010 duration. ${ }^{6,7}$ In a long-term open-label extension study, $30 \%$ of patients treated with tamsulosin reported abnormal ejaculation. ${ }^{8}$ In contrast, incidences of abnormal ejaculation related to the use of non-selective $\alpha$-blockers, such as doxazosin, terazosin or alfuzosin, generally were lower than $1.5 \% .^{3}$

The Medical Dictionary for Regulatory Activities (MedDRA), the source for standard terminology for adverse events recorded in clinical trials, uses 'retrograde ejaculation' (RE) as an umbrella term to describe a broad spectrum of patient-reported events of abnormal ejaculation, including absence of seminal emission, reduced ejaculate volume and reduced ejaculation force. This terminology reflects the formerly common belief that abnormal ejaculation was mechanistically the result of RE. It was assumed that pronounced relaxation of the bladder neck muscle caused by blockade of $\alpha_{1 \mathrm{a}}$-receptors in this area would lead to backflow of seminal fluid from the prostatic urethra into the bladder. In contrast, contraction of the bladder neck during orgasm and rhythmic contraction of the bulbospongiosus muscle facilitate propulsive ejaculation in the normal antegrade manner. A recent placebo-controlled study that examined the effects of tamsulosin and the non-selective $\alpha$-blocker alfuzosin on ejaculatory function in healthy volunteers found that tamsulosin $0.8 \mathrm{mg}$ per day caused markedly reduced ejaculate volume in $90 \%$ of patients and 
anejaculation in $35 \%$ of patients. However, analysis of post-climactic urine samples showed no increase in sperm counts, suggesting that RE did not occur. ${ }^{9}$ $\alpha_{1 a}$-Receptors are abundant not only in the bladder neck but also in the vas deferens and seminal vesicle. Recently, it has been postulated that $\alpha_{1 \mathrm{a}}$-selective $\alpha$-blockers cause reduced or absent seminal emission by inhibiting smooth muscle contraction in these genital tissues. ${ }^{10}$

Silodosin is a newly approved $\alpha$-blocker with unique receptor subtype and tissue selectivity. ${ }^{11-13}$ Compared with tamsulosin, which has in vitro selectivities of 9.55 for human $\alpha_{1 \mathrm{a}}$ versus $\alpha_{1 \mathrm{~b}}$ and $\sim 2.5$ for $\alpha_{1 \mathrm{a}}$ versus $\alpha_{1 \mathrm{~d}}$, silodosin is 162 times more selective for $\alpha_{1 \mathrm{a}}$ than for $\alpha_{1 \mathrm{~b}}$ and is $\sim 55$ times more selective for $\alpha_{1 \mathrm{a}}$ than for $\alpha_{1 \mathrm{~d}} \cdot{ }^{14}$ In two 12-week randomized, placebo-controlled, multicenter US studies, silodosin significantly improved $\mathrm{BPH}-$ related LUTS and peak urinary flow rate (Qmax). ${ }^{15}$ Results were consistent with the findings of clinical studies of silodosin in Japanese patients. ${ }^{12,16,17}$ Japanese and US phase 3 studies of silodosin reported high incidences of abnormal ejaculation. Overall, 28\% of silodosin-treated patients in the two US studies reported abnormal ejaculation (classified as RE), as did $22.3 \%$ of silodosin-treated patients in the Japanese study. ${ }^{16}$ A recent study in healthy volunteers suggests that silodosin, similar to tamsulosin, induces the absence of seminal emission rather than true RE. ${ }^{18}$

If we assume that the efficacy of silodosin and its propensity to cause abnormal ejaculation are both attributable to the selective blockade of $\alpha_{1 \mathrm{a}}$-receptors, it is reasonable to hypothesize that treated patients who experience abnormal ejaculation may achieve greater symptom relief than those with no ejaculatory disturbances. In this post hoc analysis of data from two placebocontrolled US phase 3 trials of silodosin, we examined the relationship between clinical efficacy and the absence or presence of RE.

\section{Materials and methods}

\section{Patients and treatment}

This is a post hoc analysis of combined data from two double-blind, placebo-controlled phase 3 studies (SI04009 and SI04010) (ClinicalTrials.gov identifiers NCT00224107 and NCT00224120) of silodosin in patients with BPH-related LUTS. ${ }^{15}$ Studies received Institutional Review Board (IRB) approval. Eligibility criteria and patient demographics have been described. ${ }^{15}$ In brief, patients were men aged $\geqslant 50$ years with International Prostate Symptom Score (IPSS) $\geqslant 13$, with Qmax between 4 and $15 \mathrm{ml} \mathrm{s}^{-1}$, voided volume $\geqslant 125 \mathrm{ml}$, and post-void residual urine volume $<250 \mathrm{ml}$. Patients $(N=923)$ received 12 weeks of once-daily treatment with silodosin $8 \mathrm{mg}$ or placebo. The primary efficacy end point was change from baseline in IPSS. IPSS and quality of life questionnaires were completed at weeks 0, 0.5 (day 3 or 4), 1, 2, 4 and 12; Qmax was assessed at week 0, at 2-6 h after the first dose, and at weeks 1, 2, 4 and 12.

Adverse events, reported spontaneously by the patient or observed by the investigator, were recorded and coded using the terminology of the MedDRA. All adverse events were elicited from patients using an open-ended question, such as 'Since your last visit, have you noticed any changes to your health?' The MedDRA term 'RE' was applied to ejaculatory dysfunction events described in the case report forms as 'orgasm, no semen,' 'orgasm, semen quantity reduced,' 'orgasm, semen force reduced' or 'RE' (verbatim). Worsening RE conditions were recorded separately.

\section{Assessments and statistical analyses}

In this post hoc analysis, baseline characteristics and efficacy data were stratified by ejaculation status among patients treated with silodosin (that is, absence (RE-) or presence $(\mathrm{RE}+)$ of $\mathrm{RE})$. Pair-wise comparisons of treatment effects were conducted for patients receiving placebo versus each RE-stratified silodosin treatment group and for the silodosin RE + versus the silodosin RE- group.

To evaluate the relationship between ejaculation status and silodosin efficacy, two responder analyses were performed. For the first analysis, a patient was considered a responder if he experienced a 30\% improvement in both IPSS score and Qmax from baseline to the last assessment. For the second analysis, a patient was considered a responder if he experienced improvement in Qmax of $\geqslant 3 \mathrm{ml} \mathrm{s}^{-1}$ and in IPSS total score of $\geqslant 3$ points.

For all comparisons, a significance level of 5\% was applied, with no adjustments for multiple comparisons. Baseline characteristics were compared using analysis of variance for continuous variables and a logistic model for categorical variables with two levels. Pairwise comparison of efficacy parameters was performed by analysis of covariance with baseline as covariate. Responder analysis was performed using a logistic regression model with no covariate. All $P$-values and 95\% confidence intervals for odds ratios were derived from the logistic model for assessment of group equivalency; if the odds ratio of 1 was contained by the $95 \%$ confidence interval, responses in the compared groups were considered not statistically different.

\section{Results}

Of 457 patients in the placebo group, only four $(0.9 \%)$ reported RE. Of 466 patients in the silodosin groups, 131 (28\%) reported RE (silodosin RE + group), whereas 335 $(72 \%)$ did not (silodosin RE- group); most RE events (110 of $134 ; 82 \%$ ) were reported as orgasm with no seminal emission (Table 1). Reports of RE and treatment discontinuation as a result of RE were age dependent. The mean age of patients in the silodosin $\mathrm{RE}+$ group was significantly lower than that of patients in the silodosin RE - group $(P<0.0001$; Table 2$)$. Of 150 patients younger than 60 years who were treated with silodosin, $69(46.0 \%)$ reported $\mathrm{RE}$ and $7(4.7 \%)$ discontinued because of RE. Of 191 patients aged 60-70 years, 48 $(25.1 \%)$ reported $\mathrm{RE}$ and $6(3.1 \%)$ discontinued, and of 125 patients older than 70 years, $14(11.2 \%)$ reported RE and none discontinued. Except for age, patients receiving silodosin who reported RE and those who did not receive silodosin had similar baseline characteristics (Table 2). 
Table 1 Incidence of retrograde ejaculation in two silodosin phase 3 studies by MedDRA subterms

\begin{tabular}{|c|c|c|}
\hline \multirow[t]{2}{*}{ MedDRA preferred subterm } & \multicolumn{2}{|c|}{$\begin{array}{l}\text { No. (percentage) of patients, } \\
\text { No. of events }\end{array}$} \\
\hline & $\begin{array}{l}\text { Placebo } \\
(\mathrm{n}=457)\end{array}$ & $\begin{array}{l}\text { Silodosin } \\
(\mathrm{n}=466)\end{array}$ \\
\hline Orgasm, no semen & $3(0.7), 3$ & $107(23.0), 10$ \\
\hline Orgasm, no semen (worsening) & 0 & $2(0.4), 2$ \\
\hline Orgasm, semen quantity reduced & $1(0.2), 1$ & 15 (3.2), 15 \\
\hline $\begin{array}{l}\text { Orgasm, semen quantity reduced } \\
\text { (worsening) }\end{array}$ & 0 & $6(1.3), 6$ \\
\hline $\begin{array}{l}\text { Orgasm, semen force reduced } \\
\text { (worsening) }\end{array}$ & 0 & $1(0.2), 1$ \\
\hline Retrograde ejaculation (verbatim) & 0 & $2(0.4), 2$ \\
\hline
\end{tabular}

Abbreviation: MedDRA, Medical Dictionary for Regulatory Activities.

Table 2 Effect of retrograde ejaculation status on baseline characteristics and changes from baseline in efficacy parameters

\begin{tabular}{|c|c|c|c|}
\hline Variable, mean (s.d.) & $\begin{array}{l}\text { Placebo } \\
(\mathrm{n}=457)\end{array}$ & $\begin{array}{l}\text { Silodosin RE- } \\
\quad(\mathrm{n}=335)\end{array}$ & $\begin{array}{l}\text { Silodosin RE+ } \\
\quad(\mathrm{n}=131)\end{array}$ \\
\hline \multicolumn{4}{|l|}{ Baseline data } \\
\hline Age, vears & $64.7(8.06)$ & $66.1(8.20)$ & $60.8(6.28)^{\mathrm{a}, \mathrm{b}}$ \\
\hline BMI, $\mathrm{kg} \mathrm{m}^{-2}$ & $28.5(4.30)$ & & $28.6(4.51)$ \\
\hline IPSS total score & 21.3( & 21.0 & $22.2(5.01)$ \\
\hline IPSS i & 9.3 & & 9.4 \\
\hline IPSS obstructive & $12.0(3.53)$ & $11.7(3.59)$ & $12.8(3.38)$ \\
\hline $\mathrm{nls}^{-1}$ & & & \\
\hline QoI & $4.0(1.07)$ & 3.9 & .05) \\
\hline \multicolumn{4}{|c|}{ Changes from baseline to week 12 (LOCF) } \\
\hline IPSS total score & $-3.5(5.84)$ & $-6.1(6.36)^{c}$ & $-7.2(7.23)^{\mathrm{c}}$ \\
\hline subscore & $-1.4(2.66)$ & $-2.2(2.84)^{\mathrm{c}}$ & $-2.6(3.15)^{\mathrm{c}}$ \\
\hline $\begin{array}{l}\text { IPSS obstructive } \\
\text { subscore }\end{array}$ & $-2.1(3.76)$ & $-3.8(4.19)^{\mathrm{c}}$ & $-4.6(4.59)^{\mathrm{c}}$ \\
\hline Qmax, $\mathrm{mls}^{-1}$ & $1.5(4.36)$ & $2.4(4.29)^{\mathrm{d}}$ & $3.1(4.76)^{\mathrm{d}}$ \\
\hline QoL & $-0.4(1.14)$ & $-0.8(1.29)^{\mathrm{c}}$ & $-1.0(1.35)^{\mathrm{c}}$ \\
\hline
\end{tabular}

Abbreviations: BMI, body mass index; IPSS, International Prostate Symptom Score; LOCF, last observation carried forward; Qmax, peak urinary flow rate; QoL, quality of life; RE, retrograde ejaculation.

${ }^{\mathrm{a}} P<0.0001$ versus silodosin $\mathrm{RE}-$.

${ }^{\mathrm{b}} P<0.001$ versus placebo.

${ }^{c} P<0.0001$ versus placebo.

${ }^{\mathrm{d}} P<0.02$ versus placebo.

Over the 12-week study period, irrespective of ejaculation status, patients receiving silodosin versus placebo experienced significant improvement in IPSS, including total score and irritative and obstructive subscores $(P<0.0001)$, in Qmax $(P<0.02)$ and in quality of life $(P<0.0001)$. Clinical improvements assessed by these efficacy parameters were numerically greater in patients with RE than in those without RE, but differences between the silodosin RE + and RE- groups were not statistically significant (Table 2, Figure 1).

To further analyze the relationship between ejaculation status and efficacy, two responder analyses were performed (see Materials and methods). The first analysis found that, of the patients who received placebo, 9.2\% achieved an improvement of at least $30 \%$ in both IPSS and Qmax. In comparison, $20.9 \%$ of patients in the silodosin RE- group and $27.5 \%$ in the silodosin RE+ group achieved at least $30 \%$ improvement in both IPSS and Qmax (Figure 2). The second responder analysis found that total IPSS improved by at least 3 points and
Qmax by at least $3 \mathrm{mls}^{-1}$ (3 units of improvement) in $12.9 \%$ of patients receiving placebo compared with $23.0 \%$ in the silodosin RE- group and $34.4 \%$ in the silodosin $\mathrm{RE}+$ group (Figure 2).

The odds ratios versus placebo of achieving $30 \%$ improvement were 2.61 for patients in the silodosin REgroup $(P<0.0001)$ and 3.74 for patients in the silodosin $\mathrm{RE}+$ group $(P<0.0001)$. The odds of $30 \%$ improvement for patients in the silodosin $\mathrm{RE}+$ group were 1.43 times those for patients in the silodosin RE- group $(P=0.1285)$ (Table 3 ). The odds ratios versus placebo of achieving improvement of 3 units were $2.01(P<0.001)$ for patients in the silodosin RE- group and $3.53(P<0.0001)$ for patients in the silodosin $\mathrm{RE}+$ group. The odds of achieving improvement of 3 units for patients in the silodosin RE + group were 1.75 times those for patients in the silodosin RE- group, for a statistically significant difference $(P=0.0127)$ (Table 3$)$.

\section{Discussion}

This post hoc analysis of combined data from two US phase 3 studies of silodosin revealed no major differences in baseline parameters between patients who experienced RE during 12 weeks of silodosin treatment and those who did not, with the notable exception of age, which was significantly different between the two groups. Thus, age may have predictive value for the occurrence of silodosinrelated abnormal ejaculation. However, the observation that patients who reported abnormal ejaculation were younger than those who did not probably is merely a reflection of the greater likelihood and frequency of sexual activity in younger men.

Silodosin previously has been shown to promote significant improvement in IPSS total score, with the mean decrease from baseline to study end exceeding 6 points and a mean drug-attributable effect (versus placebo) of $\sim 3$ points. ${ }^{15}$ An improvement in IPSS of 2 points is the minimum effect noticeable by patients as global symptom improvement. ${ }^{19}$ Thus, treatment with silodosin resulted in overall symptom improvement that was three times the minimum noticeable effect, and the drug-attributable effect itself was greater than the minimum noticeable effect. Silodosin compared with placebo promoted statistically significant improvement in quality of life. Previously published data from the combined phase 3 studies showed that over the course of the study, the percentage of patients who were delighted, pleased or mostly satisfied with their health-related quality of life increased from $6.9 \%$ to $32.0 \%$ in the silodosin group and from $7.2 \%$ to $22.5 \%$ in the placebo group. ${ }^{15}$

When changes in IPSS and Qmax were compared, silodosin-treated patients with $\mathrm{RE}$ had numerically greater improvement than those without RE, but the differences were not statistically significant. Responder analyses were done to determine (1) the proportions of patients with a minimum improvement of $30 \%$ in both IPSS and Qmax and (2) the proportions of patients with improvement of at least 3 units in both IPSS and Qmax. Results showed that the proportions of patients who achieved these levels of improvement were greater among those with RE than among those without RE. Furthermore, odds ratios indicated that patients with RE were significantly more likely than those without RE 

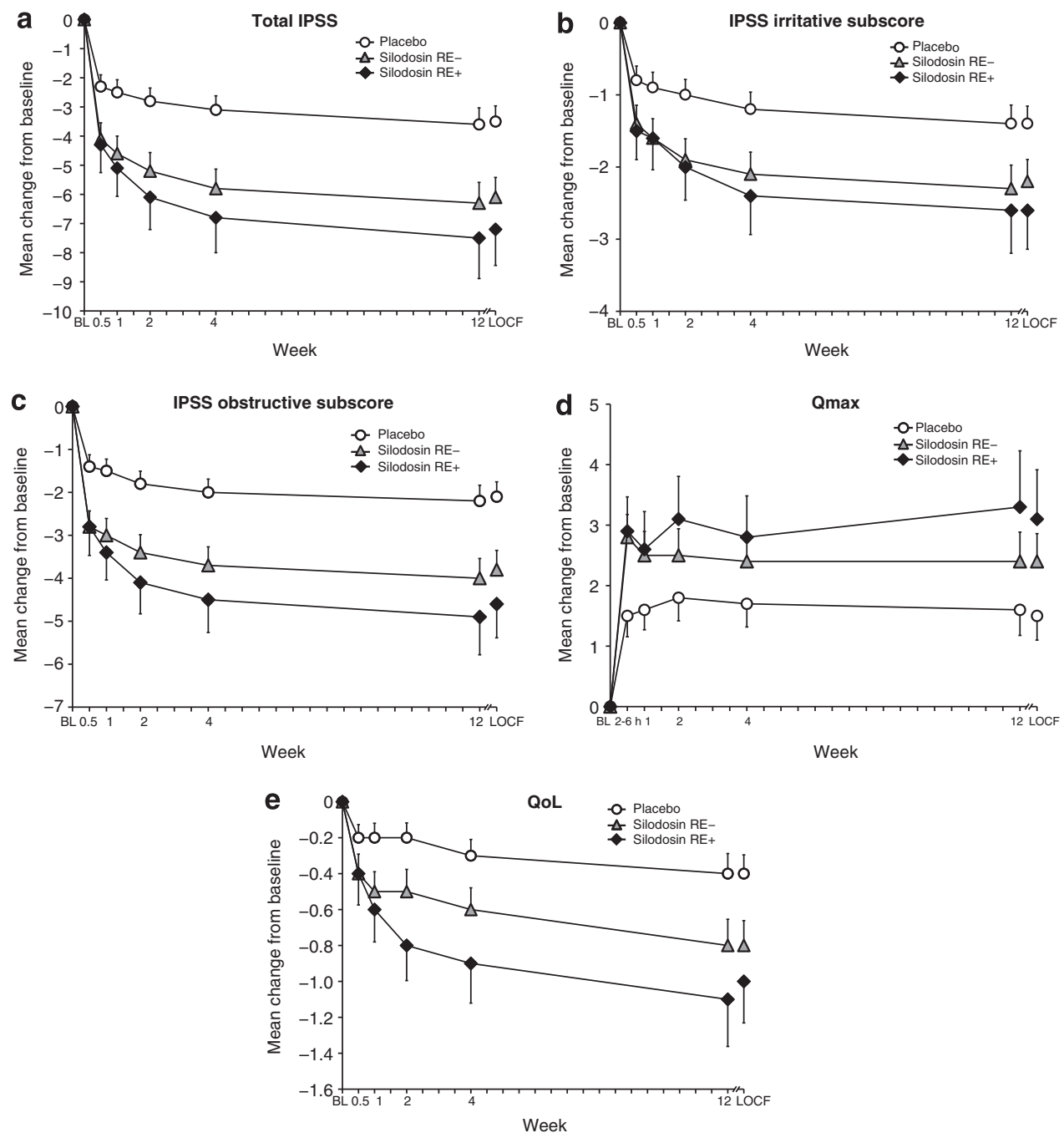

Figure 1 Mean changes from baseline in total IPSS (a), IPSS irritative subscore (b), IPSS obstructive subscore (c), Qmax (d) and QoL (e) by ejaculation status. Error bars indicate 95\% confidence intervals. Abbreviations: BL, baseline; IPSS, International Prostate Symptom Score; LOCF, last observation carried forward; Qmax, peak urinary flow rate; QoL, quality of life; RE, retrograde ejaculation.

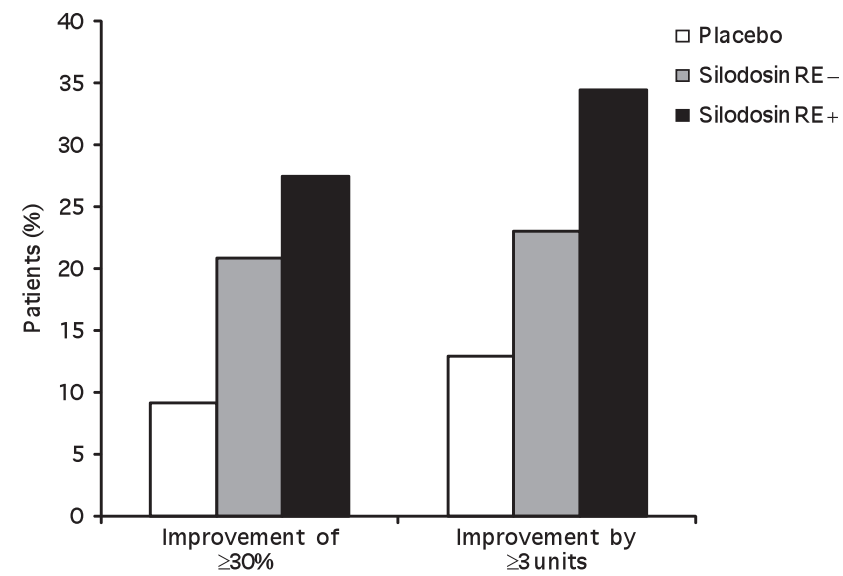

Figure 2 Patient response to silodosin treatment by ejaculation status. A patient was considered a responder if he experienced a $30 \%$ improvement in IPSS and Qmax or both an improvement in IPSS by at least 3 points and an improvement in Qmax by at least $3 \mathrm{ml} \mathrm{s}^{-1}$. Analyses were based on changes from baseline to week 12 (LOCF). IPSS, International Prostate Symptom Score; LOCF, last observation carried forward; Qmax, peak urinary flow rate; RE, retrograde ejaculation. to achieve overall symptom improvement (IPSS total by at least 3 points and Qmax improvement by at least $3 \mathrm{ml} \mathrm{s}^{-1}$ ). Compared with the placebo group, both silodosin groups showed significantly greater improvement in IPSS and Qmax in the two responder analyses.

Silodosin has been shown to be very effective in the treatment of patients with BPH-associated LUTS and to cause abnormal ejaculation in almost one-third of treated patients. ${ }^{15}$ It has been suggested that $\alpha_{1 \mathrm{a}}$-selective $\alpha$-blockers, such as silodosin, can cause absence of seminal emission by inhibiting smooth muscle contraction in genital tissues. If the results of our post hoc analysis are substantiated by future studies, the absence of seminal emission for individual patients with BPHrelated symptoms could be a sensitive indicator of a positive response to treatment with an $\alpha_{1 a}$-selective $\alpha$-blocker. Thus, ejaculatory status could help to identify men who are particularly responsive to such a therapy. However, the biological basis for lesser or greater responsiveness to therapy with $\alpha_{1 \mathrm{a}}$-selective $\alpha$-blockers remains unclear.

This post hoc analysis has important limitations. A variety of adverse events spontaneously reported by 
Table 3 Responder analyses for patients with improvement in both IPSS and Qmax from baseline to week 12 (LOCF)

\begin{tabular}{|c|c|c|c|}
\hline & Placebo $(\mathrm{n}=457)$ & Silodosin, $R E-(\mathrm{n}=335)$ & Silodosin, $R E+(\mathrm{n}=131)$ \\
\hline \multicolumn{4}{|c|}{ Improvement of $\geqslant 30 \%$ in both IPSS and Qmax from baseline to week 12 (LOCF) } \\
\hline Responders, \% (95\% CI) & $9.2(6.5,11.8)$ & $20.9(16.5,25.2)$ & $27.5(19.8,35.1)$ \\
\hline OR versus placebo $(95 \% \mathrm{CI})$ & & $\begin{array}{c}2.61(1.73,3.94) \\
P<0.0001\end{array}$ & $\begin{array}{c}3.74(2.28,6.16) \\
P<0.0001\end{array}$ \\
\hline OR versus $\mathrm{RE}-(95 \% \mathrm{CI})$ & & & $\begin{array}{c}1.43(0.90,2.28) \\
P=0.1285\end{array}$ \\
\hline \multicolumn{4}{|c|}{ Improvement by $\geqslant 3$ IPSS points and $\geqslant 3 \mathrm{ml} \mathrm{s}^{-1}$ in Qmax from baseline to week 12 (LOCF) } \\
\hline Responders, \% (95\% CI) & $12.9(9.8,16.0)$ & $23.0(18.5,27.5)$ & $34.4(26.2,42.5)$ \\
\hline OR versus placebo $(95 \% \mathrm{CI})$ & & $\begin{array}{c}2.01(1.39,2.93) \\
P=0.0002\end{array}$ & $\begin{array}{c}3.53(2.24,5.55) \\
P<0.0001\end{array}$ \\
\hline OR versus $\mathrm{RE}-(95 \% \mathrm{CI})$ & & & $\begin{array}{c}1.75(1.13,2.73) \\
P=0.0127\end{array}$ \\
\hline
\end{tabular}

Abbreviations: CI, confidence interval; IPSS, International Prostate Symptom Score; LOCF, last observation carried forward; OR, odds ratio; Qmax, peak urinary flow rate; $R E$, retrograde ejacuation.

patients were collapsed into the probably incorrect term 'RE.' Most events of RE appeared to be events of no or reduced seminal emission during orgasm. Several conditions had to be met for an event of RE to be reported: the patient had to be sexually active, had to remember the event until the next clinical visit and had to consider the event worth reporting. The observations reported in this study could be explained in part by the theoretical possibility that men with erectile dysfunction were less likely to respond to $\alpha$-blockers than were other patients; however, no evidence to support this assumption is available from the published literature. Furthermore, the number of patients who reported RE in this study was too small for the performed analyses to have sufficient statistical power. Consequently, the reported findings are preliminary and are not intended to establish a causal relationship between the efficacy of silodosin and the occurrence of RE. To substantiate a link between clinical efficacy and ejaculatory status in men treated with silodosin, prospective studies sufficiently powered to demonstrate significant differences in clinical outcomes depending on the presence or absence of seminal emission are needed. In addition, such studies should require detailed questionnaires that rigorously address sexual activity and ejaculatory function.

Results of this post hoc analysis suggest a possible relationship between silodosin efficacy and the occurrence of abnormal ejaculation as a result of treatment. This analysis showed that silodosin-treated patients who were experiencing abnormal ejaculation were significantly more likely to achieve a minimum improvement of 3 units in both IPSS and Qmax than were those with normal ejaculatory function. This observation suggests that reduced or absent seminal emission in a patient treated with silodosin may predict greater treatment efficacy. The biological basis for differences in treatment response among individual patients remains to be elucidated.

\section{Conflict of interest}

All authors have a financial and/or other relationship with Watson Laboratories.

\section{Acknowledgements}

Gary Hoel, PhD, and Lawrence A Hill, PharmD, of the Clinical Research Department at Watson Laboratories contributed to the silodosin phase 3 studies and review of this manuscript. Editorial assistance, supported by Watson Pharma, was provided by Roland Tacke, PhD, and Dolores Matthews, ELS, MEd, of Scientific Connexions. This study was supported by Watson Pharma.

\section{References}

1 Schwinn DA, Roehrborn CG. Alpha1-adrenoceptor subtypes and lower urinary tract symptoms. Int J Urol 2008; 15: 193-199.

2 Chapple CR. A comparison of varying alpha-blockers and other pharmacotherapy options for lower urinary tract symptoms. Rev Urol 2005; 7 (Suppl 4): S22-S30.

3 American Urological Association Practice Guidelines Committee. Results of the Treatment Outcomes Analyses. In: Guideline on the management of benign prostatic hyperplasia (BPH). American Urological Association Education and Research, Inc., Chapter 3, 2006. Available at: http://www.auanet.org/content/ guidelines-and-quality-care/clinical-guidelines/main-reports / bph-management/chapt_3_appendix.pdf.

4 Djavan B, Marberger M. A meta-analysis on the efficacy and tolerability of alpha1-adrenoceptor antagonists in patients with lower urinary tract symptoms suggestive of benign prostatic obstruction. Eur Urol 1999; 36: 1-13.

5 Andersson KE, Gratzke C. Pharmacology of alpha1-adrenoceptor antagonists in the lower urinary tract and central nervous system. Nat Clin Pract Urol 2007; 4: 368-378.

6 Wilt TJ, Mac DR, Rutks I. Tamsulosin for benign prostatic hyperplasia. Cochrane Database Syst Rev 2003; 1: CD002081.

7 Lepor H. Long-term evaluation of tamsulosin in benign prostatic hyperplasia: placebo-controlled, double-blind extension of phase III trial. Tamsulosin Investigator Group. Urology 1998; 51: 901-906.

8 Narayan P, Lepor H. Long-term, open-label, phase III multicenter study of tamsulosin in benign prostatic hyperplasia. Urology 2001; 57: 466-470.

9 Hellstrom WJ, Sikka SC. Effects of acute treatment with tamsulosin versus alfuzosin on ejaculatory function in normal volunteers. J Urol 2006; 176: 1529-1533.

10 Michel MC. Alpha1-adrenoceptors and ejaculatory function. Br J Pharmacol 2007; 152: 289-290.

11 Tatemichi S, Tomiyama Y, Maruyama I, Kobayashi S, Kobayashi $\mathrm{K}$, Maezawa A et al. Uroselectivity in male dogs of silodosin (KMD-3213), a novel drug for the obstructive component 
of benign prostatic hyperplasia. Neurourol Urodyn 2006; 25 792-799.

12 Yoshida M, Homma Y, Kawabe K. Silodosin, a novel selective alpha 1A-adrenoceptor selective antagonist for the treatment of benign prostatic hyperplasia. Expert Opin Investig Drugs 2007; 16: 1955-1965.

13 Kobayashi S, Tomiyama Y, Tatemichi S, Hoyano Y, Kobayashi M, Yamazaki Y. Effects of silodosin and tamsulosin on the urethra and cardiovascular system in young and old dogs with benign prostatic hyperplasia. Eur I Pharmacol 2009; 613: 135-140.

14 Tatemichi S, Kobayashi K, Maezawa A, Kobayashi M, Yamazaki Y, Shibata N. Alpha1-adrenoceptor subtype selectivity and organ specificity of silodosin (KMD-3213)]. Yakugaku Zasshi 2006; 126 (Spec no): 209-216.

15 Marks LS, Gittelman MC, Hill LA, Volinn W, Hoel G. Rapid efficacy of the highly selective alpha1A-adrenoceptor antagonist silodosin in men with signs and symptoms of benign prostatic hyperplasia: Pooled results of 2 phase 3 studies. J Urol 2009; 181: 2634-2640.

16 Kawabe K, Yoshida M, Homma Y. Silodosin, a new alpha1Aadrenoceptor-selective antagonist for treating benign prostatic hyperplasia: results of a phase III randomized, placebo- controlled, double-blind study in Japanese men. BJU Int 2006; 98: 1019-1024.

17 Takao T, Tsujimura A, Kiuchi H, Matsuoka Y, Miyagawa Y, Nonomura $\mathrm{N}$ et al. Early efficacy of silodosin in patients with lower urinary tract symptoms suggestive of benign prostatic hyperplasia. Int J Urol 2008; 15: 992-996.

18 Kobayashi K, Masumori N, Hisasue S, Kato R, Hashimoto K, Itoh $\mathrm{N}$ et al. Inhibition of seminal emission is the main cause of anejaculation induced by a new highly selective alpha1Ablocker in normal volunteers. I Sex Med 2008; 5: 2185-2190.

19 Barry MJ, Williford WO, Chang Y, Machi M, Jones KM, WalkerCorkery $\mathrm{E}$ et al. Benign prostatic hyperplasia specific health status measures in clinical research: how much change in the American Urological Association symptom index and the benign prostatic hyperplasia impact index is perceptible to patients? J Urol 1995; 154: 1770-1774.

This work is licensed under the Creative SOMERIGHIS RESERE Commons Attribution-NonCommercial-No
Derivative Works 3.0 Unported License. To view a copy of this license, visit http://creativecommons.org/licenses/ by-nc-nd/3.0/ 